\title{
Clasificación de nueve sitios de bosque mixto de CONÍFERAS CON PRESENCIA DE TAXUS GLOBOSA EN LA SiERRA Madre Oriental, Nuevo León y Tamaulipas, México
}

\author{
Mario A. García-Aranda ${ }^{1,2}$, A. Eduardo Estrada-Castillón ${ }^{1}$, Cesar M. Cantú-Ayala ${ }^{1}$ y \\ Marisela PANDO-MORENO ${ }^{1}$
}

1Facultad de Ciencias Forestales, Universidad Autónoma de Nuevo León 2Autor para la correspondencia: mgarcia_20@yahoo.com.mx

\begin{abstract}
Resumen: Se muestrearon nueve sitios con bosque mixto de coníferas que presentan al tejo mexicano (Taxus globosa var. floridana) en la Sierra Madre Oriental, en los estados de Nuevo León y Tamaulipas. Se cuantificó área basal, densidad, cobertura, altura y número de especies arbóreas y arbustivas asociadas, así como las variables altitud, exposición, pendiente y pedregosidad. Los bosques estudiados fueron divididos de acuerdo con su distribución en norte y sur para su análisis y compararon mediante una prueba de $t$. Para cada sitio se calculó el índice de diversidad Shannon-Wiener y se aplicó una prueba de $t$ de Hutcheson para determinar las diferencias en diversidad entre los sitios. Se calculó el valor de importancia de cada especie por sitio y esta información fue evaluada mediante análisis de conglomerados, utilizando sólo aquellas especies con valor de importancia $\geq 5 \%$ en al menos uno de los sitios. El área basal y la altura fueron diferentes significativamente entre los sitios norte y sur, y lo mismo se observó para las variables altitud y pendiente; las demás variables no mostraron diferencias significativas con base en la prueba de $t$. La mayor diversidad se observó en los sitios inaccesibles como El Butano y los lejanos a zonas urbanas como Las Tinajas. Los sitios cuyos índices de diversidad no difirieron significativamente fueron los geográficamente cercanos entre sí. El dendrograma mostró que La Camotera y El Rumbadero, junto con Potrero Redondo, son los sitios que poseen mayor similitud. Destaca la presencia de Abies vejarii subsp. mexicana, Juglans mollis, Quercus rysophylla y Tilia mexicana en los bosques donde se encuentra Taxus globosa var. floridana.
\end{abstract}

Palabras clave: Abies vejarii subsp. mexicana, clasificación de las comunidades, Juglans mollis, Noreste de México, Quercus rysophylla, Tilia mexicana.

\begin{abstract}
Nine sites of mixed-conifer forests where the Mexican yew (Taxus globosa) inhabits in the Sierra Madre Oriental at the States of Nuevo León and Tamaulipas were sampled. We quantified the basal area, density, canopy cover, height and number of plants of the associate species, as well as altitude, topographic aspect, slope, and stoniness. The sampled sites were divided in northern and southern sites according to their distribution in the study area and their attributes were compared with $t$ tests. A Shannon-Wiener diversity index was calculated for each site and a Hutcheson $t$ test was applied to find differences in diversity values between sites. The importance value for each species per site was calculated and the information of the nine forest sites was evaluated by means of cluster analysis, using only those species with importance value of $\geq 5 \%$ in at least one site. The $t$ tests showed that both basal area and plant height were different between the northern and southern sites, as were altitude and slope; the other variables showed statistical equality. The higher diversity was found at inaccessible sites such as El Butano and in areas far away from urban zones such as Las Tinajas. The sites without differences regarding their diversity indices are geographically closer to each other. The dendrogram showed that La Camotera and El Rumbadero, along with Potrero had the highest similarity. Abies vejarii subsp. mexicana, Juglans mollis, Quercus rysophylla and Tilia mexicana are the most common species in the forests where Taxus globosa var. floridana inhabitat.
\end{abstract}

Key words: Abies vejarii subsp. mexicana, community classification, Juglans mollis, Northeastern Mexico, Quercus rysophylla Tilia mexicana.

$\mathbf{E}_{\mathrm{d}}^{1}$ género Taxus se compone de 24 especies y 55 variedades en todo el mundo (Spjut, 2007). Este autor reconoce que las subespecies T. globosa Schltdl. var. globosa y
T. globosa Schltdl. var. floridana (Nutt. ex Champ.) Spjut. se distribuyen en México, siendo T. globosa var. floridana la subespecie presente en Nuevo León, Tamaulipas y Vera- 
cruz (Spjut, 2007). En Europa el tejo es una planta de lento crecimiento, tolerante a la sombra (Dov iak, 2003). Su importancia en la extracción de fitoquímicos útiles en tratamientos contra el cáncer es también conocida (Shemluck et al., 2003; Soto-Hernández et al., 2005). La presencia de taxol en $T$. globosa es comparable a los niveles encontrados en especies europeas (Soto-Hernández et al., 2005). A pesar de ello, aún existen pocos estudios sobre la especie en territorio mexicano y específicamente en la región noreste, lo que justifica su estudio en un intento por aportar información sobre su ecología encaminada a un manejo sostenible de la especie. En México, T. globosa tiene una distribución discontinua y restringida a microhábitats particulares y es considerada como rara en las categorías oficiales de conservación de especies (García y Castillo, 2000). Se conocen poblaciones de $T$. globosa en el sureste del país, en el estado de Chiapas, específicamente en laderas húmedas con bosque de Pinus y bosque de Abies (Contreras y Luna, 2001), y en el área central de México en la zona montañosa del municipio de Rioverde, San Luis Potosí (García y Castillo, 2000). Las poblaciones de T. globosa var. floridana en el noreste de México se han observado en el estado de Tamaulipas en el bosque mesófilo de montaña de la Reserva de El Cielo, Gómez Farías, y en Nuevo León, en las comunidades boscosas de Picea-Abies cercanas a La Trinidad, así como en bosques mixtos. Sin embargo, se desconoce qué otras especies están asociadas a T. globosa var. floridana, especie común bajo del dosel de estos bosques, y si existe una diferenciación entre sus poblaciones que tienen distribución norte o sur. A nivel de semillas, se ha observado que las poblaciones del norte de México varían entre regiones geográficas, siendo las del norte de mayor tamaño que las de las poblaciones del centro (Ramírez et al., 2011). Las características particulares del hábitat de las subespecies de $T$. globosa en la región noreste de México son aún poco conocidas. No se tiene registro de las especies que coexisten con T. globosa var. floridana en esta área de su distribución, así como las variables bióticas y abióticas mayormente asociadas con su presencia. El propósito de este estudio fue clasificar las comunidades vegetales donde han sido encontradas poblaciones de T. globosa var. floridana mediante un análisis cuantitativo de variables bióticas y abióticas para conocer cuáles son las principales especies con las que se asocia.

\section{Materiales y métodos}

Área de estudio. El área de estudio se localiza en los estados de Nuevo León y Tamaulipas, en la Sierra Madre Oriental, entre las coordenadas geográficas $23^{\circ} 02$ ' $31^{\prime \prime}-25^{\circ} 19^{\prime} 56^{\prime \prime}$ $\mathrm{N}$ y $98^{\circ} 57^{\prime} 39.5^{\prime \prime}-100^{\circ} 14^{\prime} 58^{\prime \prime} \mathrm{O}$ (Figura 1). Esta zona se ubica en la subprovincia de la Gran Sierra Plegada dentro de la provincia fisiográfica de la Sierra Madre Oriental. La presencia de la especie en la zona noreste se encuentra muy asociada con el clima tipo $\mathrm{C}(\mathrm{w})$ templado y subhúme-

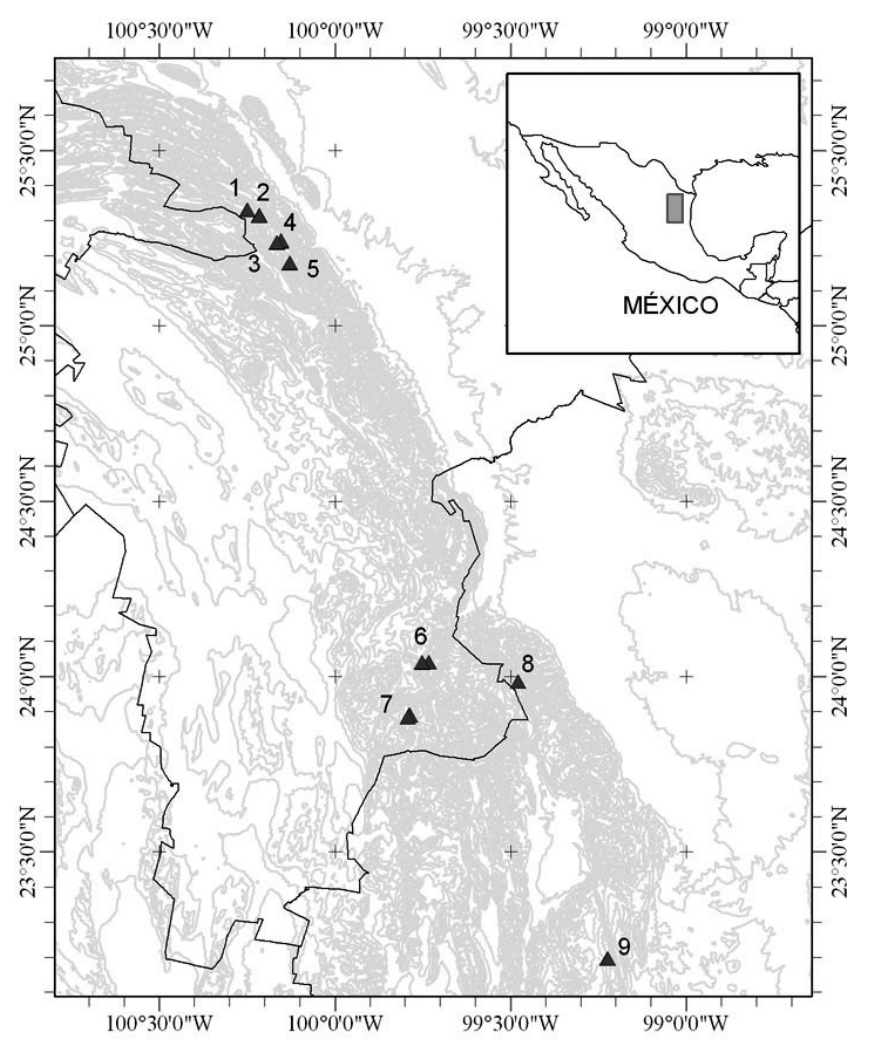

Figura 1. Área de estudio: 1. El Tejocote, Santiago, N. L.; 2. La Camotera, Santiago, N. L.; 3. Potrero Redondo, Santiago, N. L.; 4. Cañón El Rumbadero, Montemorelos, N. L.; 5. El Butano, Montemorelos, N. L.; 6. Agua Fría, Aramberri, N. L.; 7. Las Tinajas, Gral. Zaragoza, N. L.; 8. Puerto Purificación, Hidalgo, Tamaulipas; 9. Julilo, Jaumave, Tamaulipas.

do. Los suelos predominantes en las áreas con presencia de Taxus globosa var. floridana son los litosoles y los tipos de vegetación predominantes son los bosques de pino, pino-encino y encino-pino (INEGI, 1986). La ecorregión principal de la zona es la de los bosques de encino-pino de la Sierra Madre Oriental (Olson y Dinerstein, 2002).

Mediciones en campo. Se ubicaron y muestrearon nueve sitios de bosque mixto donde se localiza Taxus globosa var. floridana (Figura 1), siete de ellos en el estado de Nuevo León y dos en el estado de Tamaulipas. En cada sitio se establecieron cuadros de $20 \times 20 \mathrm{~m}$, dependiendo del tamaño de cada población de Taxus. Para Nuevo León se establecieron 35 cuadros en los siete sitios y cinco para los dos sitios de Tamaulipas.

En cada uno de los 40 cuadros se registró el número de especies arbóreas y arbustivas presentes, se midió la altura total de cada individuo registrado, se midió su diámetro con el uso de forcípula, se calculó la cobertura aérea de la copa registrando largo y ancho y usando la fórmula Cobertura $=(\operatorname{largo}+$ ancho/4) $2 \cdot \Phi($ Mueller-Dombois y Ellenberg, 
Cuadro 1. Resultados de prueba de $t$ en dos bloques (norte y sur) de variables en 40 cuadros con presencia de Taxus globosa var. floridana. ${ }^{*}=$ significativo al $95.0 \%,{ }^{* *}=$ significativo al $99.0 \%, \mathrm{~ns}=\mathrm{no}$ significativo.

\begin{tabular}{|c|c|c|}
\hline Variable & $\begin{array}{l}\text { Valor crítico } \\
\text { de } \mathrm{t} \text { (dos colas) }\end{array}$ & $P$ \\
\hline Área basal & 2.040 & $0.023^{*}$ \\
\hline Altura & 2.028 & $0.052 *$ \\
\hline Cobertura & 2.040 & $0.100 \mathrm{~ns}$ \\
\hline Densidad & 2.110 & $0.095 \mathrm{~ns}$ \\
\hline$\%$ Cobertura de hierbas & 2.797 & $0.00008^{* *}$ \\
\hline No. de especies arbóreas y arbustivas & 2.052 & $0.0770 \mathrm{~ns}$ \\
\hline
\end{tabular}

1974), el área basal se calculó con la fórmula $=\varpi($ dap $) 2 / 4$ (Young, 1991), se estimó de manera visual la cobertura de plantas herbáceas y se calculó la densidad por hectárea tanto de Taxus globosa var. floridana como de las especies arbóreas y arbustivas presentes en cada cuadro. Con base en una serie de repeticiones hechas en cada sitio, se obtuvieron los valores relativos de cobertura, densidad y frecuencia y el valor de importancia (V.I.). Además, para cada cuadro se registraron la altitud (con GPS), la pendiente y la exposición con el uso de una brújula-clinómetro, y se estimó de manera visual el porcentaje de pedregosidad.

Se determinó si había diferencias estadísticas en los datos de número de especies arbóreas y arbustivas acompañantes, altura, cobertura aérea, área basal, densidad, cobertura de hierbas; así como también de las variables abióticas altitud, exposición, pendiente y pedregosidad para los nueve sitios. Para ello, la información obtenida fue organizada en bases de datos como una matriz considerando dos bloques para hacer una prueba de $t$ con el supuesto de varianzas desiguales debido a que las poblaciones de Taxus globosa var. floridana localizadas en el noreste de México se encuentran separadas en un grupo compacto de cinco sitios ubicados en el extremo norte y otro grupo más disperso de cuatro sitios en la zona sur del área de estudio (Figura 1), habiendo entre éstos una distancia de $150 \mathrm{~km}$, lo cual supone una discontinuidad en su distribución existiendo una posible alopatría (Papavero et al., 1994; en Espinosa-Organista et al., 2002)

Cuadro 2. Resultados de pruebas de $t$ en dos bloques (norte y sur) de variables abióticas en 40 cuadrantes con presencia de Taxus globosa var. floridana. ${ }^{* * *}=$ significativo con $P<0.001, \mathrm{~ns}=$ no significativo.

\begin{tabular}{lcc}
\hline Variable & $\begin{array}{c}\text { Valor crítico } \\
\text { de t (dos colas) }\end{array}$ & $P$ \\
\hline Altitud & 3.725 & $0.00037^{* * *}$ \\
Exposición & 2.045 & $0.71003 \mathrm{~ns}$ \\
Pendiente & 3.922 & $0.00660^{* * *}$ \\
\% Cobertura pedregosidad & 2.037 & $0.85800 \mathrm{~ns}$
\end{tabular}

entre los sitios ubicados en la zona norte y los sitios de la zona sur del área de estudio (Apéndices 2, 3).

Análisis de diversidad. Se utilizó el índice de diversidad de Shannon-Wiener (Shannon, 1948), para los nueve sitios de muestreo y para determinar si había diferencias significativas de diversidad entre sitios se hicieron pruebas pareadas entre los nueve sitios utilizando la prueba de $t$ propuesta por Hutcheson $\left(t=H_{1}^{\prime}-H_{2}{ }_{2} / S_{H 1}-S_{H 2}\right)$, donde $H i$ representa el valor del índice de diversidad del sitio y $\mathrm{S}_{H i}$ representa la varianza en la diversidad del sitio, Hutcheson (1970).

Clasificación. Para hacer la clasificación de los nueve sitios, se integró una matriz con los V.I. $\geq 5 \%$ en al menos un sitio de las especies arbóreas y arbustivas presentes en los nueve sitios (Apéndice 1). Se utilizó el programa MVSP ver. 3.1 (Kovach, 1999), usando una técnica jerárquica politética aglomerativa (Gauch, 1982) mediante el procedimiento de varianza mínima (Ward, 1963). Para la construcción de matrices y clasificación de los sitios de bosque se aplicó el Índice de Similitud de Sørensen y la técnica de WPGMA (Mueller-Dumbois y Ellenberg, 1974), respectivamente.

\section{Resultados}

Comparación de sitios. Las pruebas de $t$ realizadas para las variables de la comunidad indican que el área basal $\left(t_{(0.05 ; 30)}\right.$ $=2.04, P=0.02)$, la altura de las plantas $\left(t_{(0.05 ; 36)}=2.02, P\right.$ $=0.05) \mathrm{y}$ el porcentaje de cobertura de plantas herbáceas $\left(t_{(0.05 ; 31)}=2.797, P=0.00008\right)$ fueron significativamente diferentes entre los sitios norte y sur, mientras que la cobertura, la densidad y el número de especies arbóreas y arbustivas no difirieron entre los dos grupos (Cuadro 1). Con relación a las variables abióticas, la altitud $\left(t_{(0.001 ; 25)}=3.725, P=0.00037\right)$ y la pendiente $\left(t_{(0.001 ; 18)}=3.922, P=0.0066\right)$ mostraron diferencias significativas entre los dos grupos y el resto de las variables no mostró diferencias significativas entre los grupos norte y sur (Cuadro 2).

Análisis de diversidad. El Butano (2.02) fue el sitio con mayor índice de diversidad de Shannon -Wiener, seguido por Las Tinajas, (1.82), mientras que El Tejocote, (0.98) y La Camotera. (0.98) son los sitios con el valor de diversidad más bajo (Cuadro 3). Los valores de Equitatividad $(J)$ tuvieron un comportamiento similar a los calculados para diversidad (Cuadro 3). La prueba $t$ de Hutcheson mostró diferencias significativas en la mayoría de los sitios $(P<$ 0.05), con excepción de los pares de sitios La Camotera-El Tejocote $(t=0.003$ (2) 0.05), Potrero Redondo-Aramberri $(t$ $=0.398$, (2) 0.05 , Potrero Redondo-Puerto Purificación $(t=$ 0.145 , (2) 0.05$)$, El Rumbadero-Zaragoza $(t=-1.968$, (2) 0.05$)$, Aramberri-Puerto Purificación $(t=-0.177$, (2) 0.05) $\mathrm{y}$ JuliloPuerto Purificación [ $t=1.943$, (2) 0.05) , Cuadro 4]. 
Cuadro 3. Índices de diversidad para nueve sitios con presencia de Taxus globosa var. floridana

\begin{tabular}{|c|c|c|c|c|}
\hline Sitio & $\begin{array}{l}\text { No. } \\
\text { taxa }\end{array}$ & $\mathrm{N}$ & $\begin{array}{c}\text { Shannon } \\
\mathrm{H}\end{array}$ & $\begin{array}{c}\text { Equitatividad } \\
\mathrm{J}\end{array}$ \\
\hline El Butano, Montemorelos, N.L. & 9 & 58 & 2.02 & 0.879 \\
\hline Las Tinajas, Zaragoza, N.L. & 10 & 425 & 1.82 & 0.789 \\
\hline El Rumbadero, Montemorelos, N.L. & 11 & 142 & 1.64 & 0.714 \\
\hline Julilo, Jaumave, Tamps. & 9 & 197 & 1.49 & 0.646 \\
\hline Potrero Redondo, Santiago, N.L. & 8 & 198 & 1.30 & 0.565 \\
\hline Puerto Purificación, Hidalgo Tamps. & 11 & 309 & 1.29 & 0.560 \\
\hline Agua Fría, Aramberri, N.L. & 8 & 442 & 1.27 & 0.553 \\
\hline La Camotera, Santiago, N.L. & 9 & 454 & 0.98 & 0.425 \\
\hline El Tejocote, Santiago, N.L. & 5 & 78 & 0.98 & 0.425 \\
\hline
\end{tabular}

Clasificación. El análisis de conglomerados reveló la existencia de dos grupos y un sitio aislado (Figura 2) a un nivel de corte de 0.34. Los dos grupos estuvieron conformados por cuatro sitios cada uno. El grupo 1, el más compacto de los tres, está formado por dos subgrupos: en el subgrupo 1A, los sitios El Rumbadero (RUM) y La Camotera (CAM) son los sitios más afines, pues comparten entre sí seis especies (Abies vejarii subsp. mexicana, Croton cortesianus, Juglans mollis, Pinus pseudostrobus, Quercus rysophylla y Tilia mexicana), con un coeficiente de similitud de Sørensen de 0.73; a estos dos sitios se les une el sitio Potrero Redondo (POT), compartiendo con ambos las especies J. mollis, $P$. pseudostrobus, $Q$. rysophylla y $T$. mexicana, con un coeficiente de similitud de 0.62; finalmente se une el subgrupo 1B, Puerto Purificación (PPU), que comparte sólo una es- pecie con el resto de los sitios del grupo (T. mexicana), de ahí su bajo nivel de similitud (0.42) con los restantes sitios de este grupo.

El grupo 2 está formado por dos subgrupos (Figura 2). En el subgrupo 2C, los sitios El Tejocote (TEJ) y El Butano (BUT) son los más afines, ya que comparten tres especies (Abies vejarii subsp. mexicana, Juglans mollis y Quercus polymorpha) con una índice de similitud de 0.57; a este subgrupo se une el sitio de Agua Fría (ARA) que comparte con el anterior dos especies (A. vejarii subsp. mexicana y J. mollis), con un coeficiente de similitud de 0.49; Las Tinajas (ZAR, 2D), se agrupa al resto de los subgrupos con un coeficiente de similitud de 0.38 , pues comparte con el resto de estos subgrupos sólo una especie, A. vejarii subsp. mexicana. El sitio 3, Julilo (JUL), fue el más disímil, con un coeficiente de similitud de 0.27 , compartiendo sólo, $A$. vejarii subsp. mexicana con el resto de los sitios.

\section{Discusión}

Las diferencias encontradas en altura, área basal y porcentaje de cobertura de las hierbas encontradas entre los sitios norte y sur se pueden atribuir en parte a la presión de uso del suelo que hay sobre los bosques, ya que éstos están ubicados dentro del Parque Nacional Cumbres de Monterrey, el cual tiene una afluencia continua de visitantes. Esta situación somete a los sitios del norte a una mayor presión que a los sitios del sur, los cuales están más dispersos y menos comunicados. Por este efecto se observa que los sitios del norte presentan menor altura y área basal que los sitios del sur, además de tener una mayor cobertura de plantas herbáceas. En cambio, la cobertura aérea, la densidad y el número

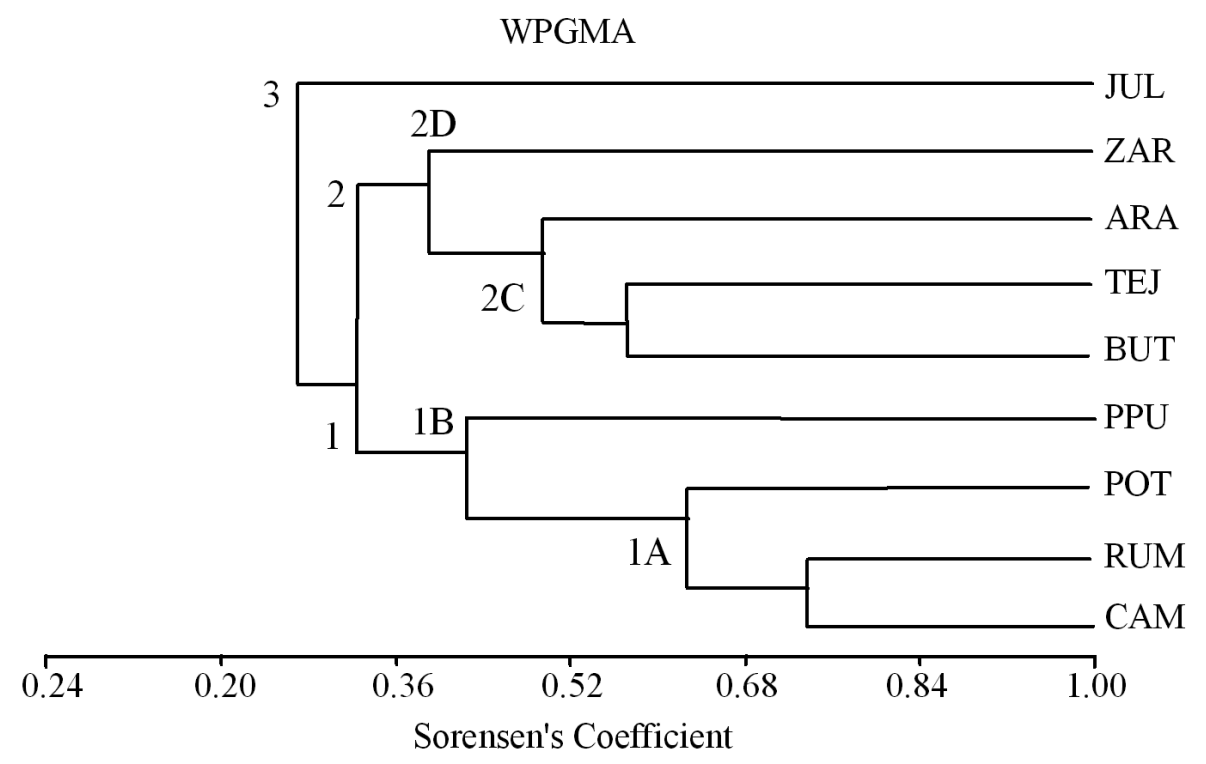

Figura 2. Clasificación de los nueve sitios con base en los valores de importancia de las especies asociadas con Taxus globosa var. floridana del noreste de México. 
Cuadro 4. Valores de $t$ de la prueba pareada de Hutcheson (parte inferior izquierda de la matriz) y grados de libertad (parte superior derecha de la matriz) para la comparación entre índices de diversidad de nueve sitios con presencia de Taxus globosa var. floridana; * $=$ Significativo, ns = no significativo (2) 0.05. La Camotera (CAM), Potrero Redondo (POT), El Butano (BUT), El Tejocote (TEJ), El Rumbadero (RUM), Agua Fría, Aramberri (ARA), Las Tinajas, Zaragoza (ZAR), Julilo (JUL), Puerto Purificación (PPU).

\begin{tabular}{lccccccccc}
\hline & CAM & POT & BUT & TEJ & RUM & ARA & ZAR & JUL & PPU \\
\hline CAM & - & 575.55 & 155.35 & 159.43 & 312.31 & 831.76 & 791.85 & 455.32 & 658.13 \\
POT & $-4.016^{*}$ & - & 135.63 & 144.70 & 269.37 & 441.86 & 407.15 & 369.19 & 506.26 \\
BUT & $-11.383^{*}$ & $-8.041^{*}$ & - & 135.58 & 178.78 & 107.80 & 99.52 & 183.42 & 199.50 \\
TEJ & $0.003 \mathrm{~ns}$ & $3.145^{*}$ & $9.336^{*}$ & - & 189.00 & 120.48 & 113.61 & 187.87 & 198.00 \\
RUM & $-6.776^{*}$ & $-3.551^{*}$ & $3.590^{*}$ & $-5.676^{*}$ & - & 234.19 & 219.56 & 318.80 & 361.46 \\
ARA & $-4.054^{*}$ & $0.398 \mathrm{~ns}$ & $9.036^{*}$ & $-3.047^{*}$ & $4.111^{*}$ & - & 863.64 & 344.55 & 526.41 \\
ZAR & $-11.836^{*}$ & $-7.560^{*}$ & $2.539^{*}$ & $-8.782^{*}$ & $-1.968 \mathrm{~ns}$ & $-9.217^{*}$ & - & 321.87 & 495.23 \\
JUL & $-5.464^{*}$ & $-2.032^{*}$ & $5.311^{*}$ & $-4.501^{*}$ & $1.468^{*}$ & $2.525^{*}$ & $4.005^{*}$ & - & 479.93 \\
PPU & $-3.347^{*}$ & $0.145 \mathrm{~ns}$ & $7.290^{*}$ & $-2.756^{*}$ & $3.328^{*}$ & $-0.177 \mathrm{~ns}$ & $6.432^{*}$ & $1.943 \mathrm{~ns}$ & - \\
\hline
\end{tabular}

de especies arbóreas y arbustivas presentes fueron similares (Cuadro 1). Aunque los sitios del norte se ubican en terrenos de menor altitud y pendiente, la exposición barlovento que aporta humedad por orografía y exposición directa en temporadas de lluvia (Rzedowski, 1981) y la pedregosidad no presentaron diferencias significativas.

Los sitios con mayor diversidad corresponden a zonas de poca accesibilidad por su topografía, como es el caso de El Butano y El Rumbadero, o por su lejanía de zonas ampliamente urbanizadas, como es el caso de Las Tinajas y Julilo. En contraste, los sitios El Tejocote y La Camotera tuvieron valores bajos de diversidad y equitatividad, posiblemente por la cercanía a la zona urbanizada de Monterrey a pesar de estar dentro del ANP Cumbres de Monterrey. La prueba t de Hutcheson aplicada para comparar los índices de diversidad a los nueve sitios indica que existe una variación en la diversidad en 30 de las 36 combinaciones entre los nueve sitios. Entre los sitios cuyos valores de diversidad no difirieron destacan las comparaciones entre La Camotera-El Tejocote, El Rumbadero-Zaragoza, Aramberri-Puerto Purificación y Julilo-Puerto Purificación que son geográficamente cercanos y comparten condiciones ambientales similares. Por el contrario, las comparaciones Potrero Redondo-Aramberri y Potrero Redondo-Puerto Purificación mostraron igualdad entre sus valores de diversidad aunque son sitios distantes entre sí.

El dendrograma mostró que los sitios muy cercanos geográficamente tienen mayor similitud sólo para El Rumbadero, la Camotera y Potrero Redondo. El sitio Julilo tiene la menor similitud con el resto de los sitios, lo que es consistente con su posición geográfica en el extremo sur del área de estudio, en una zona que representa el límite norte en la distribución del bosque mesófilo de montaña, donde Taxus globosa var. floridana coexiste con especies como Fraxinus sp., Magnolia tamaulipana y Ternstroemia sylvatica. Los sitios inconsistentes en relación a su similitud y cercanía geográfica son Puerto Purificación (PPU) que se agrupa al complejo de sitios del norte y El Tejocote-El Butano 2C que se agrupó al complejo de sitios del sector sur.

Se concluye que el hábitat de Taxus globosa var. floridana se caracteriza por ubicarse principalmente en áreas de exposición noreste a elevaciones entre 1,400 hasta los 2,690 m s.n.m., en zonas de bosques mixtos con presencia de especies arbóreas como Abies vejarii subsp. mexicana, Juglans mollis, Quercus rysophylla y Tilia mexicana.

Dentro de la región del noreste de México, los sitios con Taxus del sector más sureño presentan una mayor área basal, altura de arbolado y menor cobertura de hierbas que los sitios del norte que se encuentran más perturbados. Por sus características topográficas, los sitios del sur se ubican a mayores elevaciones, con una pendiente mayor que los sitios ubicados en el norte. Estas diferencias presentes a lo largo de la distribución, aunadas a los factores asociados al disturbio han tenido efecto en la fitodiversidad presente en cada uno de los sitios. Notoriamente, Julilo, el sitio más lejano e inaccesible tiene diferencias con ocho de los nueve sitios, siendo similar en fitodiversidad únicamente a Puerto Purificación, un sitio geográficamente cercano.

\section{Agradecimientos}

A Dino Ulises González Uribe por sus sugerencias y revisión de los análisis estadísticos; a Rafael Cruz Alonso y Antonio Conde Vázquez por su apoyo en el muestreo de campo.

\section{Literatura citada}

Contreras-Medina R. y Luna I. 2001. Presencia de Taxus globosa Schltdl. (Taxaceae) en el estado de Chiapas, México. Polibotánica 12:51-56.

Dov iak M. 2003. Population dynamics of the endangered English 
yew (Taxus baccata L.) and its management implications for biosphere reserves of the Western Carpathians. Final report on Young Scientist Award 2002 research study UNESCO-MAB, Paris

Espinosa-Organista D., Morrone J.J., Llorente-Bousquets J. y Flores-Villela O. 2002. Introducción al Análisis de Patrones en Biogeografía Histórica. Universidad Nacional Autónoma de México-Facultad de Ciencias, México, D.F.

García S.F. y Castillo P.L. 2000. Aspectos ecológicos de Taxus globosa Schltdl. en las Mesas de San Isidro, Municipio de Rioverde, San Luis Potosí. Biotam 11:11-18.

Gauch H.G. 1982. Multivariate Analysis in Community Ecology. Cambridge University Press, Cambridge.

Hutcheson K. 1970. A test for comparing diversities based on the Shannon formula. Journal of Theoretical Biology 29:151-154.

INEGI [Instituto Nacional de Estadística, Geografía e Informática]. 1986. Síntesis geográfica del estado de Nuevo León. Instituto Nacional de Estadística, Geografía e Informática-Secretaría de Programación y Presupuesto, México D.F.

Kovach W.L. 1999. MVSP - Multivariate Statistical Package, v.3.1. Kovach Computing Services, Pentreath.

Mueller-Dombois D. y Ellenberg H. 1974. Aims and Methods of Vegetation Ecology. Wiley. Nueva York.

Olson D.M. y Dinerstein E. 2002. The Global 2000: priority ecoregions for global conservation. Annals of the Missouri Botanical
Garden 89:199-224.

Ramírez-Sánchez S.E., López-Upton J., García-de los Santos G., Vargas-Hernández J.J., Hernández-Livera A. y Ayala-Garay O.J. 2011. Variación morfológica de semillas de Taxus globosa Schltdl. provenientes de dos regiones geográficas de México. Revista Fitotecnia Mexicana 34:93-99.

Rzedowski J. 1981. Vegetación de México. Limusa, México, D.F.

Shannon C.E. 1948. A mathematical theory of communication. Bell System Technical Journal 27:379-423, 623-656.

Shemluck M.J., Estrada E., Nicholson R. y Brobst S.W. 2003. A preliminary study of the taxane chemistry and natural history of the Mexican yew, Taxus globosa Schltdl. Boletín de la Sociedad Botánica de México 72:119-127.

Soto-Hernández M., Sanjurjo M., González-Garza M.T., Cruz-Vega D. y Giral-González F. 2005. El tejo mexicano (Taxus globosa Schltdl.). Potencial de su aprovechamiento en taxol. Ciencia Ergo Sum 7:277-279.

Spjut R.W. 2007. Taxonomy and nomenclature of Taxus (TAXACEAE). Journal of Botany Reserach Institute of Texas 1:203289.

Ward J.H. 1963. Hierarchical grouping to optimize an objective function. Journal of the American Statistical Association 58:236-244.

Young R.A. 1991. Introducción a las Ciencias Forestales. Limusa, México, D.F.

Recibido: 31 de julio de 2010

Aceptado: 24 de octubre 2011 
Apéndice 1. Matriz de especies con V. I. $\geq 5 \%$ en al menos uno de los nueve sitios con presencia de Taxus globosa var. floridana. La Camotera (CAM), Potrero Redondo (POT), El Butano (BUT), El Tejocote (TEJ), Cañón El Rumbadero (RUM), Agua Fría (ARA), Las Tinajas (ZAR), Julilo (JUL) y Puerto Purificación (PPU).

\begin{tabular}{|c|c|c|c|c|c|c|c|c|c|}
\hline Especie / Sitio & CAM & POT & BUT & TEJ & RUM & ARA & ZAR & $J U L$ & PPU \\
\hline Abies vejarii subsp. mexicana (Martínez) Farjon & 3.09 & - & 33.83 & 99.09 & 33.21 & 74.04 & 40.51 & 8.37 & 9.76 \\
\hline Agave sp. & & & & & 10.22 & 8.27 & & - & - \\
\hline Arbutus xalapensis Kunth & - & - & - & - & - & 15.15 & 16.00 & - & - \\
\hline Asplenium resiliens Kunze & - & 4.12 & - & - & - & - & - & - & - \\
\hline Brahea dulcis Mart. & - & - & - & - & - & - & - & 4.26 & - \\
\hline Carpinus caroliniana Walter & - & - & 18.90 & - & - & 8.07 & - & - & - \\
\hline Carya illinoinensis (Wangenh.) K.Koch & - & - & 8.16 & - & - & - & - & - & - \\
\hline $\begin{array}{l}\text { Carya ovata var. mexicana (Engelm.exHemsl.) } \\
\text { W.E.Manning }\end{array}$ & - & - & - & - & - & - & - & 16.20 & 20.78 \\
\hline Celtis laevigata Spreng. & - & - & - & - & 22.16 & - & - & - & - \\
\hline Chamaedorea radicalis Mart. & - & - & - & - & - & - & - & 7.81 & - \\
\hline Cornus excelsa Kunth & - & - & - & - & - & - & - & 5.39 & - \\
\hline Cornus florida L. & - & 62.14 & - & - & - & - & - & - & 20.51 \\
\hline Crataegus greggiana Eggl. & - & - & 3.67 & - & - & - & - & - & 4.30 \\
\hline Croton fruticulosus Tott. & 6.38 & - & - & 9.09 & - & - & - & - & - \\
\hline Croton cortesianus Kunth & 88.45 & - & - & - & 3.54 & 7.60 & - & 3.49 & 22.25 \\
\hline Cupressus arizonica Greene & 6.39 & - & 5.86 & - & 11.02 & - & - & 8.16 & - \\
\hline Dasylirion texanum Scheele & - & - & - & - & 8.62 & - & - & - & - \\
\hline Fraxinus sp. & - & - & - & - & - & - & - & 14.88 & - \\
\hline Garrya laurifolia Hartw. ex Benth. & - & - & - & - & - & - & 18.98 & - & - \\
\hline Garrya ovata Benth. & 7.69 & - & - & - & 11.51 & - & - & - & - \\
\hline Ilex discolor Hemsl. & - & - & - & - & - & - & - & 18.32 & - \\
\hline Ilex ораса Ait. & - & - & 16.97 & - & - & - & - & - & - \\
\hline Ilex rubra S.Watson & - & - & - & - & - & 24.63 & - & - & - \\
\hline Ilex sp. & - & - & - & - & - & - & - & - & 12.05 \\
\hline Juglans mollis Engelm. & 33.66 & 26.95 & 19.72 & 41.44 & 67.09 & 4.64 & - & - & - \\
\hline Juniperus deppeana Steud. & - & - & - & 9.55 & - & - & 2.13 & - & - \\
\hline Liquidambar styraciflua L. & - & - & - & - & - & - & - & - & 10.34 \\
\hline Lithospermum gracile Raf. & - & - & 40.58 & - & - & - & - & - & - \\
\hline Litsea pringlei Bartlett & - & 33.89 & - & - & 3.56 & - & - & - & 5.31 \\
\hline Magnolia tamaulipana A.Vázquez & - & - & - & - & - & - & - & 23.76 & - \\
\hline Malacomeles denticulata (Kunth) G.N.Jones & - & - & - & - & - & - & 4.37 & - & - \\
\hline Nectandra sanguinea Rol. & - & - & - & - & - & - & - & 6.63 & - \\
\hline Ostrya virginiana Britton, Sterns \& Poggenb. & - & 8.30 & - & - & - & 33.53 & - & - & 11.12 \\
\hline Persea podadenia S.F.Blake & - & - & - & - & - & - & - & 6.79 & - \\
\hline Picea martinezii T.F.Patterson & - & - & 35.08 & - & - & 72.81 & 10.84 & - & - \\
\hline Pinus flexilis E.James & - & - & - & - & - & - & 14.86 & - & - \\
\hline Pinus patula Schltdl. \& Cham & - & - & - & - & - & - & - & - & 20.41 \\
\hline Pinus pseudostrobus Lindl. & 30.29 & 6.19 & - & - & 8.49 & - & - & - & - \\
\hline Platanus mexicana Moric. & - & - & - & 15.54 & - & - & - & - & - \\
\hline Prunus serotina Ehrh. & 10.07 & - & - & - & - & - & - & - & - \\
\hline
\end{tabular}


Mario A. García-ARanda et aL.

Apéndice 1. Continuación

\begin{tabular}{lccccccccc}
\hline Especie / Sitio & CAM & POT & BUT & TEJ & RUM & ARA & ZAR & JUL & PPU \\
\hline Pseudotsuga menziesii (Mirb.) Franco & - & - & - & - & - & - & 7.90 & - & - \\
Quercus canbyi Trel. & - & - & - & - & 13.05 & - & 72.25 & - & 10.46 \\
Quercus mexicana Benth. & - & - & 15.63 & - & - & - & 16.25 & 27.60 & - \\
Quercus polymorpha Schltdl. \& Cham. & - & - & 35.11 & 9.87 & - & - & 23.02 & - & - \\
Quercus rysophylla Weath. & 64.37 & 88.29 & - & - & 3.95 & - & 5.23 & - & - \\
Quercus sp. & - & - & - & - & - & 11.87 & - & - & - \\
Salix lasiolepis Benth. & 3.11 & - & - & - & - & - & - & - & - \\
Taxus globosa Schltdl. & 37.34 & 66.10 & 43.93 & 115.41 & 94.29 & 39.39 & 67.66 & 61.88 & 147.28 \\
Ternstroemia sylvatica Cham. \& Schltdl. & - & - & - & - & - & - & - & 70.04 & - \\
Tilia mexicana Schltdl. & 9.16 & 4.02 & 22.56 & - & 9.29 & - & - & 7.77 & 5.43 \\
Wimmeria concolor Cham. \& Schltdl. & - & - & - & - & - & - & - & 8.65 & - \\
\hline
\end{tabular}


Apéndice 2. Valores de la media por cuadro para las variables bióticas en nueve sitios con presencia de Taxus globosa var. floridana. En los grupos norte $(\mathrm{N})$ y sur $(\mathrm{S})$

\begin{tabular}{|c|c|c|c|c|c|c|c|}
\hline Sitio (grupo) & & $\begin{array}{c}\text { Área basal } \\
\left(\mathrm{m}^{2}\right)\end{array}$ & $\begin{array}{c}\text { Altura } \\
(\mathrm{m})\end{array}$ & $\begin{array}{c}\text { Cobertura } \\
\text { ha }\end{array}$ & $\begin{array}{c}\text { Densidad } \\
\text { ha }\end{array}$ & $\begin{array}{c}\text { Cobertura } \\
\text { de hierbas } \\
(\%)\end{array}$ & $\begin{array}{c}\text { No. de taxa } \\
\text { arbóreos y } \\
\text { arbustivos }\end{array}$ \\
\hline \multirow[t]{5}{*}{ La Camotera $(\mathrm{N})$} & 1 & 22.50 & 5.56 & $9,277.53$ & 15,625 & 35.00 & 6 \\
\hline & 2 & 12.21 & 6.75 & $8,070.75$ & 23,125 & 40.00 & 8 \\
\hline & 3 & 15.57 & 5.15 & $13,269.50$ & 31,875 & 25.00 & 8 \\
\hline & 4 & 34.40 & 5.59 & $23,586.00$ & 146,250 & 80.00 & 8 \\
\hline & 5 & 26.01 & 7.08 & $23,362.45$ & 79,375 & 85.00 & 6 \\
\hline \multirow[t]{6}{*}{ Potrero Redondo (N) } & 1 & 2.50 & 3.50 & $3,305.45$ & 7,500 & 10.0 & 4 \\
\hline & 2 & 8.41 & 4.95 & $15,680.02$ & 30,000 & 30.0 & 5 \\
\hline & 3 & 11.10 & 7.57 & $9,801.57$ & 23,750 & 10.0 & 8 \\
\hline & 4 & 6.93 & 5.04 & $12,967.12$ & 28,125 & 5.0 & 6 \\
\hline & 5 & 9.14 & 7.68 & $17,524.88$ & 47,500 & 30.0 & 6 \\
\hline & 6 & 20.85 & 10.60 & $18,790.26$ & 11,250 & 5.0 & 3 \\
\hline \multirow[t]{5}{*}{ El Butano $(\mathrm{N})$} & 1 & 16.61 & 7.87 & $12,763.11$ & 23,125 & 34.3 & 8 \\
\hline & 2 & 24.80 & 5.84 & $20,015.48$ & 63,750 & 41.3 & 6 \\
\hline & 3 & 56.52 & 8.20 & $33,035.22$ & 33,125 & 70.0 & 11 \\
\hline & 4 & 24.82 & 6.50 & $22,603.56$ & 31,250 & 70.0 & 11 \\
\hline & 5 & 38.05 & 7.80 & $35,937.46$ & 51,875 & 60.0 & 8 \\
\hline \multirow[t]{3}{*}{ El Tejocote (N) } & 1 & 10.58 & 4.60 & $11,602.49$ & 14,375 & 70.0 & 5 \\
\hline & 2 & 17.34 & 9.07 & $20,254.63$ & 20,000 & 70.0 & 4 \\
\hline & 3 & 7.14 & 4.85 & $7,449.31$ & 16,250 & 70.0 & 5 \\
\hline \multirow[t]{5}{*}{ Cañón El Rumbadero (N) } & 1 & 1.23 & 3.29 & 7,397.08 & 23,125 & 5.0 & 11 \\
\hline & 2 & 1.82 & 4.97 & $11,217.65$ & 19,375 & 10.0 & 6 \\
\hline & 3 & 1.63 & 4.16 & $10,353.71$ & 14,375 & 0.0 & 4 \\
\hline & 4 & 14.55 & 6.56 & $12,104.16$ & 18,125 & 5.0 & 6 \\
\hline & 5 & 26.83 & 5.36 & $9,012.15$ & 24,375 & 10.0 & 8 \\
\hline \multirow[t]{4}{*}{ Agua Fría (S) } & 1 & 17.78 & 6.31 & $20,730.98$ & 51,250 & 10.0 & 8 \\
\hline & 2 & 21.02 & 8.37 & $38,398.51$ & 110,000 & 5.0 & 7 \\
\hline & 3 & 30.43 & 7.02 & $21,201.82$ & 87,500 & 15.0 & 7 \\
\hline & 4 & 11.03 & 8.49 & $11,566.36$ & 39,375 & 15.0 & 7 \\
\hline \multirow[t]{7}{*}{ Las Tinajas (S) } & 1 & 39.47 & 7.21 & $21,721.36$ & 43,750 & 5.0 & 8 \\
\hline & 2 & 22.11 & 6.66 & $11,248.28$ & 43,125 & 5.0 & 10 \\
\hline & 3 & 27.35 & 7.69 & $13,622.93$ & 35,625 & 5.0 & 6 \\
\hline & 4 & 60.61 & 9.76 & $36,868.95$ & 440,000 & 5.0 & 6 \\
\hline & 5 & 21.29 & 5.67 & $16,770.80$ & 43,125 & 5.0 & 4 \\
\hline & 6 & 10.26 & 5.53 & $9,494.09$ & 34,375 & 5.0 & 5 \\
\hline & 7 & 18.15 & 4.96 & $16,506.81$ & 48,750 & 5.0 & 7 \\
\hline \multirow[t]{3}{*}{ Julilo (S) } & 1 & 43.84 & 9.61 & $20,021.71$ & 53,125 & 10.0 & 12 \\
\hline & 2 & 12.96 & 8.31 & $14,600.61$ & 31,250 & 5.0 & 10 \\
\hline & 3 & 41.99 & 8.30 & $27,478.11$ & 53,125 & 15.0 & 15 \\
\hline \multirow[t]{2}{*}{ Puerto Purificación (S) } & 1 & 34.16 & 6.65 & $14,588.77$ & 90,625 & 10.0 & 9 \\
\hline & 2 & 29.89 & 5.26 & $23,655.60$ & 46,250 & 10.0 & 11 \\
\hline
\end{tabular}


Apéndice 3. Altitud, exposición, pendiente y pedregosidad para cada cuadro en nueve sitios con presencia de Taxus globosa var. floridana en los grupos norte $(\mathrm{N})$ y sur $(\mathrm{S})$.

\begin{tabular}{|c|c|c|c|c|c|}
\hline Sitio (grupo) & & $\begin{array}{c}\text { Altitud } \\
\text { (m s.n.m.) }\end{array}$ & $\begin{array}{l}\text { Exposición } \\
\text { (grados) }\end{array}$ & $\begin{array}{l}\text { Pendiente } \\
\text { (grados) }\end{array}$ & $\begin{array}{c}\text { Pedregosidad } \\
(\%)\end{array}$ \\
\hline \multirow[t]{5}{*}{ La Camotera (N) } & 1 & 1,598 & -1.00 & 0.0 & 73.33 \\
\hline & 2 & 1,561 & 260.00 & 15.0 & 55.00 \\
\hline & 3 & 1,523 & -1.00 & 2.0 & 70.00 \\
\hline & 4 & 1,523 & 310.00 & 5.7 & 70.00 \\
\hline & 5 & 1,574 & 310.00 & 5.7 & 30.00 \\
\hline \multirow[t]{6}{*}{ Potrero Redondo (N) } & 1 & 1,417 & 340.00 & 6.0 & 40.0 \\
\hline & 2 & 1,400 & 355.00 & 6.0 & 20.0 \\
\hline & 3 & 1,421 & 8.00 & 12.0 & 25.0 \\
\hline & 4 & 1,442 & 1.00 & 12.0 & 30.0 \\
\hline & 5 & 1,456 & 32.00 & 5.0 & 29.0 \\
\hline & 6 & 1,414 & 338.00 & 6.0 & 30.0 \\
\hline \multirow[t]{5}{*}{ El Butano (N) } & 1 & 2,148 & 356.00 & 10.3 & 25.0 \\
\hline & 2 & 2,122 & 330.00 & 7.0 & 20.0 \\
\hline & 3 & 2,181 & 297.00 & 15.0 & 10.0 \\
\hline & 4 & 2,165 & 328.00 & 12.0 & 10.0 \\
\hline & 5 & 2,131 & 144.00 & 7.0 & 15.0 \\
\hline \multirow[t]{3}{*}{ El Tejocote $(\mathrm{N})$} & 1 & 1,936 & 287.00 & 5.0 & 3.0 \\
\hline & 2 & 1,928 & 279.00 & 8.0 & 3.0 \\
\hline & 3 & 1,949 & 1.00 & 6.0 & 3.0 \\
\hline \multirow[t]{5}{*}{ Cañón El Rumbadero (N) } & 1 & 1,774 & 90.00 & 10.0 & 70.0 \\
\hline & 2 & 1,794 & 90.00 & 8.0 & 65.0 \\
\hline & 3 & 1,795 & 90.00 & 15.0 & 70.0 \\
\hline & 4 & 1,790 & 90.00 & 10.0 & 75.0 \\
\hline & 5 & 1,786 & 90.00 & 6.0 & 70.0 \\
\hline \multirow[t]{4}{*}{ Agua Fría (S) } & 1 & 2,200 & 359.00 & 20.0 & 60.0 \\
\hline & 2 & 2,209 & 359.00 & 20.0 & 75.0 \\
\hline & 3 & 2,207 & 303.00 & 25.0 & 60.0 \\
\hline & 4 & 2,205 & 330.00 & 35.0 & 70.0 \\
\hline \multirow[t]{7}{*}{ Las Tinajas (S) } & 1 & 2,547 & 352.00 & 15.0 & 50.0 \\
\hline & 2 & 2,533 & 352.00 & 20.0 & 50.0 \\
\hline & 3 & 2,537 & 338.00 & 25.0 & 50.0 \\
\hline & 4 & 2,536 & 24.00 & 8.0 & 50.0 \\
\hline & 5 & 2,564 & 316.00 & 30.0 & 50.0 \\
\hline & 6 & 2,553 & 338.00 & 20.0 & 50.0 \\
\hline & 7 & 2,690 & 14.00 & 10.0 & 50.0 \\
\hline \multirow[t]{3}{*}{ Julilo (S) } & 1 & 1,618 & -1.00 & 0.0 & 0.0 \\
\hline & 2 & 1,610 & -1.00 & 0.0 & 5.0 \\
\hline & 3 & 1,590 & 2.00 & 5.0 & 2.0 \\
\hline \multirow[t]{2}{*}{ Puerto Purificación (S) } & 1 & 1,844 & 135.00 & 15.0 & 5.0 \\
\hline & 2 & 1,832 & 12.00 & 12.0 & 5.0 \\
\hline
\end{tabular}

